\title{
Nonclassical interaction-free detection of objects in a monolithic total-internal-reflection resonator
}

\author{
Harry Paul ${ }^{1}$ and Mladen Pavičić ${ }^{1,2, \dagger}$ \\ ${ }^{1}$ Max-Planck-AG Nichtklassische Strahlung, Humboldt University of Berlin, \\ D-12484 Berlin, Germany \\ ${ }^{2}$ Department of Mathematics, University of Zagreb, GF, Kačićeva 26, POB 217, \\ HR-10001 Zagreb, Croatia
}

\begin{abstract}
We show that with an efficiency exceeding $99 \%$ one can use a monolithic totalinternal-reflection resonator in order to ascertain the presence of an object without transferring a quantum of energy to it. We also propose an experiment on the probabilistic meaning of the electric field that contains only a very few photons.
\end{abstract}

PACS numbers: 42.50, 03.65.Bz

\section{INTRODUCTION}

Quantum optical measurements can exhibit an outstanding and completely nonclassical feature of detecting an object without transferring a single quantum of energy to it. For example, after the second beam splitter of a properly adjusted Mach-Zehnder interferometer one can always put a detector in such a position that it almost never detects a photon. If it does, then we are certain that an object blocked one path of the interferometer. A measurement which makes use of this quantum mechanical feature was first designed by Elitzur and Vaidman.1 1 日 For dramatic effect Elitzur and Vaidman assumed that the object is a bomb and showed that it would explode in only $50 \%$ of the tests if asymmetrical beam splitter is employed. The tests, of course, always have to be carried out with single photons. Kwiat et al. . single photons. They aim at reducing the probability of exploding the bomb to as close to $0 \%$ as possible and propose using two identical cavities weakly coupled by a highly reflective beam splitter. Due to the interference the probability for a photon inserted into the first cavity to be located in it approaches 0 , while the probability that it is found in the second cavity approaches 1 , at a certain time $T_{N}$. However, if there is an opaque object (a bomb) in the second cavity the probabilities reverse. So, if we insert a detector in the first cavity we almost never get a click if there is no absorber in the second cavity, and almost always if there is one. The probability of exploding the bomb when there is a detector in the first cavity approaches zero. The drawback of the proposal is that it is apparently very hard to 
carry it out. In particular, inserting a detector into a cavity at a given time and introducing a single photon into a cavity are, by no means, simple tasks.

In Sec. 2 we propose a very simple, very efficient, and easily feasible interaction-free experiment - with an arbitrary high probability of detecting the bomb without exploding itwhich is based on the resonance in a single monolithic total-internal-reflection resonator which has recently shown significant practical advantage and very high efficiencies. The proposal assumes a pulse laser beam or a continuous wave (cw) laser beam and a properly cut isotropic crystal. The additional advantage of the present proposal over the proposals of Kwiat et al. 3. is that the latter ones infer information on the presence or absence of the bomb in the system from the absence of a detector click while we get the information from the firing of appropriate detectors.

Actually, we will perform a classical calculation. As is well known, there is a formal correspondence between the classical and the quantum description in the sense that classical quantities, e.g., the amount of energy absorbed by a bomb, are identical to quantum mechanical ensemble averages. When the absorbed energy is, on average, only a small fraction of an energy quantum $h \nu$, this means in reality that in most cases no absorption takes place at all, and only in a few cases one photon is actually absorbed. This is a consequence of the corpuscular nature of light. In other words, interpreting classical intensities as quantum mechanical probabilities to find a photon, allows us to "translate" our classical results into quantum mechanical predictions.

Our elaboration shows that, in accordance with quantum theory, an overwhelming number of photons detected by a detector indicating a presence of a bomb in the system will not exchange any energy with the bomb. Only in rare cases a photon will transfer an energy quantum $h \nu$ to the bomb. Nevertheless, on average, there is no difference between the quantum and the classical picture. This means that many other parallels between the two pictures, such as wave amplitude calculations, coherence time and length, optical resonator calculations, cavity photon decay time, etc., should be preserved. On the other hand, in one-photon quantum interference the amplitudes of electric field have a probabilistic meaning only, and one might wonder whether a classical reasoning would give a correct answer. For example, Weinfurter et al.6 and Fearn et al7 proposed an experiment which would decide whether sudden changing of boundary conditions of frustrated downconverted photons affects photons instantaneously or after a (classically untenable) delay which would allow all parts of the system (atoms emitting downconverted photons) to "receive" the information on the changed conditions.

In Sec. 3 we propose an experiment which would decide whether sudden changing of boundary conditions imposed on photon paths (object-no object) redirect the photons (into $D_{r}$ instead into $D_{t}$ and vice versa) instantaneously (classically untenable) or after a delay which would allow for sufficiently many round trips to build up the interference.

\section{INTERACTION-FREE EXPERIMENT}

The lay-out of the experiment is shown in Fig. 1. The experiment uses an uncoated monolithic total-internal-reflection resonator (MOTIRR) coupled to two triangular prisms by the frustrated total internal reflection (FTIR). A 9 . A squared MOTIRR requires a relative

refractive index with respect to the surrounding medium $n>1.41$ in order to confine a 
beam to the resonator (the angle of incidence being $45^{\circ}$ ). If, however, another medium (in our case the right triangular prism in Fig. 1) is brought within a distance of the order of the wavelength, the total reflection within the resonator will be frustrated and a fraction of the beam will "tunnel out" from the resonator. Depending on the dimension of the gap and the polarization of the incidence beam one can well define reflectivity $R$ within the range from $10^{-5}$ to 0.99995 . 10 The main advantage of such a coupling - in comparison with coated resonators - is that the losses are extremely small: down to $0.3 \%$. In the same way a beam can "tunnel into" the resonator through the left triangular prism in Fig. 1, provided the condition $n>1.41$ is fulfilled for the prism too. The incident laser beam is chosen to be polarized perpendicularly to the incident plane so as to give a unique reflectivity for each photon. The faces of the resonator are polished spherically to give a large focusing factor. A round trip path for the beam is created in the resonator as shown in Fig. 1. A cavity is cut in the resonator and filled with an index-matching fluid to reduce losses. (Before we carry out the measurement we have to wait until the fluid comes to a standstill to avoid a possible destabilization of the phase during round trips of the beam.) Now, if there is an object in the cavity in the round trip path of the beam in the resonator, the incident beam will be almost totally reflected (into $D_{r}$ ) and if there is no object, the beam will be almost totally transmitted (into $D_{t}$ ).

We start with a formal presentation of the experiment. Our aim is first to determine the intensity of the beam reaching detector $D_{r}$ when the crystal is at resonance, and then this intensity when an opaque object (bomb) is in the round-trip path in the crystal. A portion of a polarized incoming beam of amplitude $A(\omega)$ is totally reflected from the inner surface of the left coupling prism (see Fig. 1) and suffers a phase shift which ranges from $0^{\circ}$ (for the critical angle $\theta_{1}=\arcsin n$ ) to $180^{\circ}$ (for $\theta_{1}=0^{\circ}$ ), where $\theta_{1}$ is the incident angle at the prism inner surface.11 When we couple the ring cavity (MOTIRR) to the prism, a part of the incoming beam will tunnel into MOTIRR and the reflected beam will suffer a new phase shift $\delta$ and the complex-field-reflection coefficient will read:

$r e^{i \delta}=\left[1-\frac{2 \sin \delta_{1} \sin \delta_{2}}{\cosh 2 b x-\cos \left(\delta_{1}+\delta_{2}\right)}\right] \exp \left(\frac{\sin \delta_{1} \sinh 2 b x}{\cos \delta_{1} \cosh 2 b x-\cos \delta_{2}}\right)$,

where $\delta_{1}$ and $\delta_{2}$ are the phase shifts of the waves at coupled surfaces of the prism and MOTIRR, respectively, $x$ is the gap between the prism and MOTIRR, and $b=$ $\frac{2 \pi}{\lambda_{0}} \sqrt{n_{1}^{2} \sin ^{2} \theta_{1}-n_{2}^{2}}$, where $n_{1}$ and $n_{2}$ are refraction indexes of the prism and MOTIRR, respectively, and $\lambda_{0}$ is the vacuum wavelength. Thus detector $D_{r}$ rotated at the angle $\delta$ with respect to the incoming plane will receive the incoming power of the incident beam attenuated by $|r|^{2}$.

At resonance the waves leaving the cavity after some round trips in the cavity will add up to a destructive interference so as to make the reflected power vanish. Formally, it can be described as follows. 9 The ratio of the reflected and incoming powers is

$\eta=1-\frac{c(x)}{1+\left[\frac{2 \mathcal{F}}{\pi} \sin \frac{\delta(x)+\phi}{2}\right]^{2}}$,

where $\phi$ is the total phase shift acquired by the wave during one round trip, $\mathcal{F}$ is the finesse, and $c(x)$ is the coupling given by 
$c(x)=\frac{\left(1-e^{-2 \alpha}\right)\left[1-|r(x)|^{2}\right]}{\left[1-e^{-\alpha}|r(x)|\right]^{2}}$,

where $\alpha$ is a constant describing the round-trip losses. At resonance the reflected power will vanish when MOTIRR is impedance matched, i.e., when the gap is adjusted so as to satisfy: $\left|r\left(x_{m}\right)\right|=e^{-\alpha}$. Then $c\left(x_{m}\right)=1$ and $\phi=2 N \pi-\delta$, where $N$ is an integer, so that $\eta=0$.

To understand this result we sum up the contributions originating from round trips in the resonator, to the reflected wave. The portion of the incoming beam of amplitude $A(\omega)$ reflected into plane determined by $\delta$ is described by the amplitude $B_{0}(\omega)=-A(\omega) \sqrt{R}$, where $R=|r|^{2}$ is reflectivity. The transmitted part will travel around the resonator guided by one frustrated total internal reflection (at the face next to the right prism) and by two proper total internal reflections. After a full round trip the following portion of this beam joins the directly reflected portion of the beam by tunnelling into the left prism: $B_{1}(\omega)=$ $A(\omega) \sqrt{1-R} \sqrt{R} \sqrt{1-R} e^{i \psi} . B_{2}(\omega)$ contains three frustrated total internal reflections and so on; each subsequent round trip contributes to a geometric progression which gives the reflected amplitude

$B_{n}(\omega)=A(\omega) \sqrt{R}\left\{-1+(1-R) e^{i \psi}\left[1+R e^{i \psi}+\left(R e^{i \psi}\right)^{2}+\ldots\right]\right\}=\sum_{i=0}^{n} B_{i}(\omega)$,

where $\psi=\left(\omega-\omega_{r e s}\right) T$ is the phase added by each round trip. Here $\omega$ is the frequency of the incoming beam, $T$ is the round trip time, and $\omega_{\text {res }}$ is the selection frequency corresponding to a wavelength which satisfies $\lambda=L / k$, where $L$ is the round trip length of the cavity and $k$ is an integer. At summing up the round-trip contributions we have taken into account that (because of the above condition imposed on the total phase shift $\phi$ ) all the contributions must lie in the reflected-wave plane and that their amplitudes must carry the opposite sign (to that of the reflected wave, $-A(\omega) \sqrt{R}$ ) so as to cancel out at resonance $\psi=0$.

To get a condensed insight into the physics of the experiment let us first look at plane waves $\left[A(\omega)=A_{0}\right]$. The limit of $B_{n}(\omega)$ yields the total amplitude of the reflected beam:

$B_{r}(\omega)=\lim _{n \rightarrow \infty} B_{n}(\omega)=-A_{0} \sqrt{R} \frac{1-e^{i \psi}}{1-R e^{i \psi}}$.

We see that for any $R<1$ and $\omega=\omega_{\text {res }}$, i.e., if nothing obstructs the round trip of the beam, we get no reflection at all [i.e., no response from $D_{r}$ (see Fig. 1)]. When a bomb blocks the round trip and $R$ is close to one, then we get almost a total reflection. In terms of single photons (which we can obtain by attenuating the intensity of a laser until the chance of having more than one photon at a time becomes negligible) the probability of detector $D_{r}$ reacting when there is no bomb in the system is zero. A response from $D_{r}$ means an interaction-free detection of a bomb in the system. The probability of the response is $R$, the probability of making a bomb explode by our device is $R(1-R)$, and the probability of a photon exiting into detector $D_{t}$ is $(1-R)^{2}$.

A more realistic experimental approach we achieve by looking at two possible sources of individual photons: a cw laser and a pulse laser. For a pulse laser we make use of a Gaussian wave packet $A(\omega)=A \exp \left[-\tau^{2}\left(\omega-\omega_{r e s}\right)^{2} / 2\right]$, where $\tau$ is the coherence time which obviously must be significantly longer than the round trip time $T$. For a cw laser we use $A(\omega)=A \delta\left(\omega-\omega_{\text {res }}\right)$, i.e., assume a well stabilized laser beam locked at $\left.\omega_{\text {res }}\right)$ with a negligibly small linewidth. Incident wave is described by: 
$E_{i}^{(+)}(z, t)=\int_{0}^{\infty} A(\omega) e^{i(k z-\omega t)} d \omega$

and the reflected wave by:

$E_{r}^{(+)}\left(z^{\prime}, t\right)=\int_{0}^{\infty} B(\omega) e^{i\left(k z^{\prime}-\omega t\right)} d \omega$

Energy of the incoming beam is the energy flow integrated over time:

$I_{i}=\int_{-\infty}^{\infty} E_{i}^{(+)}(z, t) E_{i}^{(-)}(z, t) d t=\int_{0}^{\infty} A(\omega) A^{*}(\omega) d \omega$.

Energy of the reflected beam is given analogously by $I_{r ; n}=\int_{0}^{\infty} B_{n}(\omega) B_{n}^{*}(\omega) d \omega$. Thus for the both types of lasers the ratio of energies $\eta$ as a function of the number of round trips $n$ is given by:

$\eta_{n}=\frac{I_{r ; n}}{I_{i}}=R\left\{1-\frac{1-R}{1+R}\left[R^{2 n}-1+2 \sum_{j=1}^{n}\left(1+R^{2 n-2 j+1}\right) R^{j-1} \Phi(j)\right]\right\}$,

where $\Phi(j)=1$ for cw lasers and $\Phi(j)=\exp \left(-j^{2} a^{-2} 4^{-1}\right)$ for pulse lasers, where $a \equiv \tau / T$. This expression is obtained by mathematical induction from the geometric progression of the intensities of the amplitudes given by Eq. (4) and a subsequent integration over wavepackets. It follows from Eq. (5) that the series with $\Phi(j)=1$ converges, so the series with $\Phi(j)=\exp \left(-j^{2} a^{-2} 4^{-1}\right)$ converges as well. For the latter $\Phi(j)$ a straight-forward calculation yields

$\lim _{n \rightarrow \infty} \eta_{n}=\frac{\int_{0}^{\infty} B_{r}(\omega) B_{r}^{*}(\omega) d \omega}{\int_{0}^{\infty} A(\omega) A^{*}(\omega) d \omega}=1-(1-R)^{2} \frac{\int_{0}^{\infty} \frac{\exp \left[-\tau^{2}\left(\omega-\omega_{\text {res }}\right)^{2}\right] d \omega}{1-2 R \cos \left[\left(\omega-\omega_{\text {res }}\right) \tau / a\right]+R^{2}}}{\int_{0}^{\infty} \exp \left[-\tau^{2}\left(\omega-\omega_{\text {res }}\right)^{2}\right] d \omega}$,

where $B_{r}(\omega)$ is from Eq. (5). In Fig. 2 the three upper curves represent three sumsobtained for three different values of $a$, respectively - that converge to values (shown as big dots) obtained from Eq. (10). The figure shows that $a$ and $n$ are closely related in the sense that the coherence length should always be long enough $(a>200)$ to allow sufficiently many round trips (at least 200).

A cw laser oscillating on a single transverse mode has the advantage of an excellent frequency stability (down to $10 \mathrm{kHz}$, and with some effort even down to $1 \mathrm{kHz}$, in the visible range) and therefore a very long coherence length (up to $300 \mathrm{~km}$ ). 12 This yields the zero intensity at detector $D_{r}$ as with plane waves above. The only disadvantage of a cw laser is that we have to modify the setup by adding a gate which determines a time window (1 ms $1 \mu \mathrm{s}<$ coherence time) within which the input beam arrives at the crystal and which allows the intensity in the cavity to build up. Then, the intensity of the beam should be lowered to make it probable for only one photon to appear within the time window. We start each testing by opening the gate, and when either $D_{r}$ or $D_{t}$ fires, or the bomb explodes, the testing is over. Of course, detectors might fail to react but this is not an essential problem because the single photon detector efficiency has already reached $85 \%$. For, this would result in a bigger time window, but the chance of activating the bomb would remain very low. In 
any case, the possibility of a $300 \mathrm{~km}$ coherence length does not leave any doubt that a real experiment can be carried out successfully. It should be emphasized that we get information on the presence or the absence of the bomb in any case from a detector click, hence we need no additional information that a photon has actually arrived at the entrance surface. This is a great advantage over the above-mentioned proposal by Kwiat et al. (1995) in which the absence of the bomb is, in fact, inferred from the absence of a detector click. When nothing happened during the exposition time (due either to the absence of a photon or detector inefficiency), the test has to be repeated.

The main disadvantage of pulse lasers is that they have mean frequency dependent on the working conditions of the laser, so each repetition of the experiment takes a considerable time to stabilize the frequency. Their advantage is that they do not require any gates.

\section{VIRTUAL-OR-REAL-PATH EXPERIMENT}

As we have seen in the previous section there is an essential difference between our proposal and the one by Kwiat et al. interrogations carried out by a "real" photon while ours is based on boundary conditions imposed on an "empty" photon path which contains no photon. Consequently, our approach does not give rise to the quantum Zeno effect as opposed to theirs.5 Nevertheless, in our experiment we can formulate a question whether the round trip path which a photon "sees" when approaching the crystal is virtual or real. The question is similar to virtual-or-realphoton question posed by Weinfurter et al 6 and Fearn et al. supported by classical formal reasoning and calculation. In the latter experiment mirrors suppress propagation of downconverted waves forming standing waves by reflecting the waves back to the crystal. As soon as one removed the mirrors and instantaneously replaced them by detectors the latter should fire triggered by the photons in the outgoing beams due to the changed boundary condition. In our experiment, changing the boundary conditions, i.e., switching on the round trip path, means allowing the round trip path to "wind up" (according to the calculation presented in Fig. 3) even when there is no single photon in the path: "the paths are real."

The experiment is presented in Fig. 4. It is a modification of the experiment shown in Fig. 1. We tune in our FTIR-MOTIRR system so as to have as big a gap between the coupling prisms and the crystal as possible (e.g., corresponding to $R=0.9999$ ). The Rochon prism $p$ is rotated so as to fully match the phase shift as its $\mathrm{O}$-wave. Therefore, when the Pockels cell is off the round-trip path is not influenced at all. When the Pockels cell is on the path is redirected through Rochon prism $p$ (as E-wave) into detector $D_{p}$. We switch on a cw laser and let it feed the system.

When the Pockels cell is on detector $D_{r}$ should fire with the probability approaching 1. When it is off detector should $D_{t}$ fire with the probability approaching 1 .

We carry out two kinds of measurement. The first kind of measurement is switching the Pockels cell on and monitoring $D_{r}$ immediately afterwards. We accommodate the intensity of the laser beam so as to have one photon in $0.1 \mathrm{~ns}$ in average. The fastest Pockels cells have reaction time down to $0.1 \mathrm{~ns}$. The time an information traveling at the speed of light needs to spread from the Pockels cell to the incoming gap can be made as high as 4 ns by choosing the biggest available crystals. The fastest detectors have reaction time of under 1 
ns. Before we switch on the Pockels cell almost only detector $D_{t}$ fires. After we switch on the Pockels cell we monitor detector $D_{r}$ and see whether it reacts instantaneously or after 4 ns.

The second kind of measurement is switching the Pockels cell from on to off and monitoring detector $D_{r}$ immediately afterwards. We lower down the intensity of the laser beam so as to have one photon in $10 \mathrm{~ns}$ in average. We calculated that for $R=0.9999$ the resonance fully establishes after $100 \mathrm{~ns}$, i.e., after that time $D_{r}$ cannot fire (almost) at all. We monitor $D_{r}$ within this $100 \mathrm{~ns}$ and see whether detector $D_{r}$ stops firing immediately or only after several firing within the first $100 \mathrm{~ns}$. We have chosen $10 \mathrm{~ns}$ in the incoming beam so as to make sure that after switching off the Pockels cell only an "empty" wave is coming to the system. A variety of the experiment would be to lower down the intensity of the laser beam further down to under one photon in $100 \mathrm{~ns}$.

\section{CONCLUSION}

In summary, we have devised a feasible and, in principle, rather simple experimental scheme for an interaction-free detection of an absorbing object (bomb) that rests on the classical behavior of a ring resonator into which light is fed. In case of resonance and in the absence of an obstacle within the cavity, a detector $D_{t}$ in the exit channel will respond with very high probability, whereas a detector $D_{r}$ placed in the light beam reflected from the entrance mirror will almost never detect a photon. When an object is introduced into the cavity, the situation completely reverses. Hence the absence or presence of the object will in any case be indicated by a detector click. This is a great advantage of our scheme, since there is no need to ensure that a photon is actually impinging on the object. Moreover, we suggest a modification of the experimental scheme that would allow to measure time delays between blocking the resonator (or undoing a blocking) and the effect it has on the detection probabilities. We apply the scheme on the Heisenberg microscope and the 'Welcher Weg' experiment in Pavičić.13

\section{ACKNOWLEDGMENTS}

One of us (M.P.) gratefully acknowledges supports of the Alexander von Humboldt Foundation and of the Ministry of Science of Croatia. He would also like to thank David W. Cohen, Department of Mathematics, Smith College, Northampton, Massachusetts for his comments on the manuscript.

†E-mail: mpavicic@faust.irb.hr; Web-page: http://m3k.grad.hr/pavicic. 


\section{REFERENCES}

1. A. C. Elitzur and L. Vaidman, "Quantum mechanical interaction-free measurements," Found. Phys., 23, 987-997 (1993).

2. L. Vaidman, "On the realization of interaction-free measurement," Quantum Opt., 6, 119-126 (1994).

3. P. Kwiat, H. Weinfurter, T. Herzog, A. Zeilinger, and M. A. Kasevich, "Interaction-free measurement," Phys. Rev. Lett. 74, 4763-4766 (1995).

4. The experiment Kwiat et al. carried out in Ref.3 as "an initial demonstration of the principle of interaction-free measurement" is, in effect, only a realization of Elitzur and Vaidman's 50\% proposall.

5. P. Kwiat, H. Weinfurter, T. Herzog, A. Zeilinger, and M. A. Kasevich, "Experimental realization of interaction-free measurements," Ann. N. Y. Acad. Sci., 755, 383-393 (1995).

6. H. Weinfurter, T. Herzog, P. Kwiat, J. G. Rarity, A. Zeilinger, and M. Żukowski, "Frustrated downconversion: Virtual or real photons?" Ann. N. Y. Acad. Sci., 755, 61-72 (1995).

7. H. Fearn, R. J. Cook, and P. W. Milonni, "Sudden replacement of a mirror by a detector in cavity QED: Are photons counted immediately?", Phys. Rev. Lett. 74, 1327-1330 (1995).

8. K. Fiedler, S. Schiller, R. Paschotta, P. Kürz, and J. Mlynek, "Highly efficient frequency doubling with a doubly resonant monolithic total-internal-reflection resonator," Opt. Lett. 18, 1786-1788 (1993).

9. S. Schiller, I. I. Yu, M. M. Fejer, and R. L. Byer, "Fused-silica monolithic total-internalreflection resonator," Opt. Lett. 17, 378-380 (1992).

10. S. Zhu, A. W. Yu, D. W. Hawly, and R. Roy, "Frustrated total internal reflection: A demonstration and review," Am. J. Phys. 54, 601-607 (1986).

11. M. Born and E. Wolf, Principles of Optics, (Pergamon, New York, 1980).

12. O. Svelto, Principles of Lasers (Plenum Press, New York, 1993), 3rd ed.

13. M. Pavičić, "Resonance Energy-Exchange-Free Detection and 'Welcher Weg' Detection," Phys. Lett. A 223, 241-245 (1996). 


\section{FIGURES}

Fig. 1. Lay-out of the proposed experiment. For the shown free round trips within the total-internal-reflection resonator the incident laser beam tunnels in and out so as to give the zero intensity of the reflected beam, i.e., detector $D_{r}$ does not react even when the incoming frustrated total reflectivity is approaching one. However, when the bomb is immersed in the (index-matching) liquid then practically the whole incoming beam reflects into $D_{r}$.

Fig. 2. Realistic values of $\eta$ [ratio of the incoming and reflected powers, given by Eq. (9)] for $R=0.98$. For pulse lasers -3 upper curves represent sums from Eq. (9) [with $\left.\Phi(j)=\exp \left(-j^{2} a^{-2} 4^{-1}\right)\right]$ as a function of $n$ for $a=100, a=200$, and $a=400$, where $a \equiv \tau / T$ is a ratio of the coherence time $\tau$ and the round-trip time $T$; dots represent the corresponding values of $\eta$ obtained from Eq. (10). For cw lasers - the lowest curve represents the sum given by Eq. (9) [with $\Phi(j)=1$ ] as a function of the number of round trips $n$.

Fig. 3. Realistic values of $\eta$ [ratio of the incoming and reflected powers, Eq. (9)] for $R=0.98$ (the lowest curve), 0.99, 0.995, 0.997, and 0.998 as functions of the number of round trips $n$.

Fig. 4. Lay-out of the proposed virtual-or-real-path experiment. When the Pockels cell $c$ is on it redirects the round-trip path through Rochon prism $p$ into detector $D_{p}$ and therefore almost only detector $D_{r}$ fires. When the Pockels cell $c$ is off there is no influence on the round-trip path and almost only detector $D_{t}$ fires. 


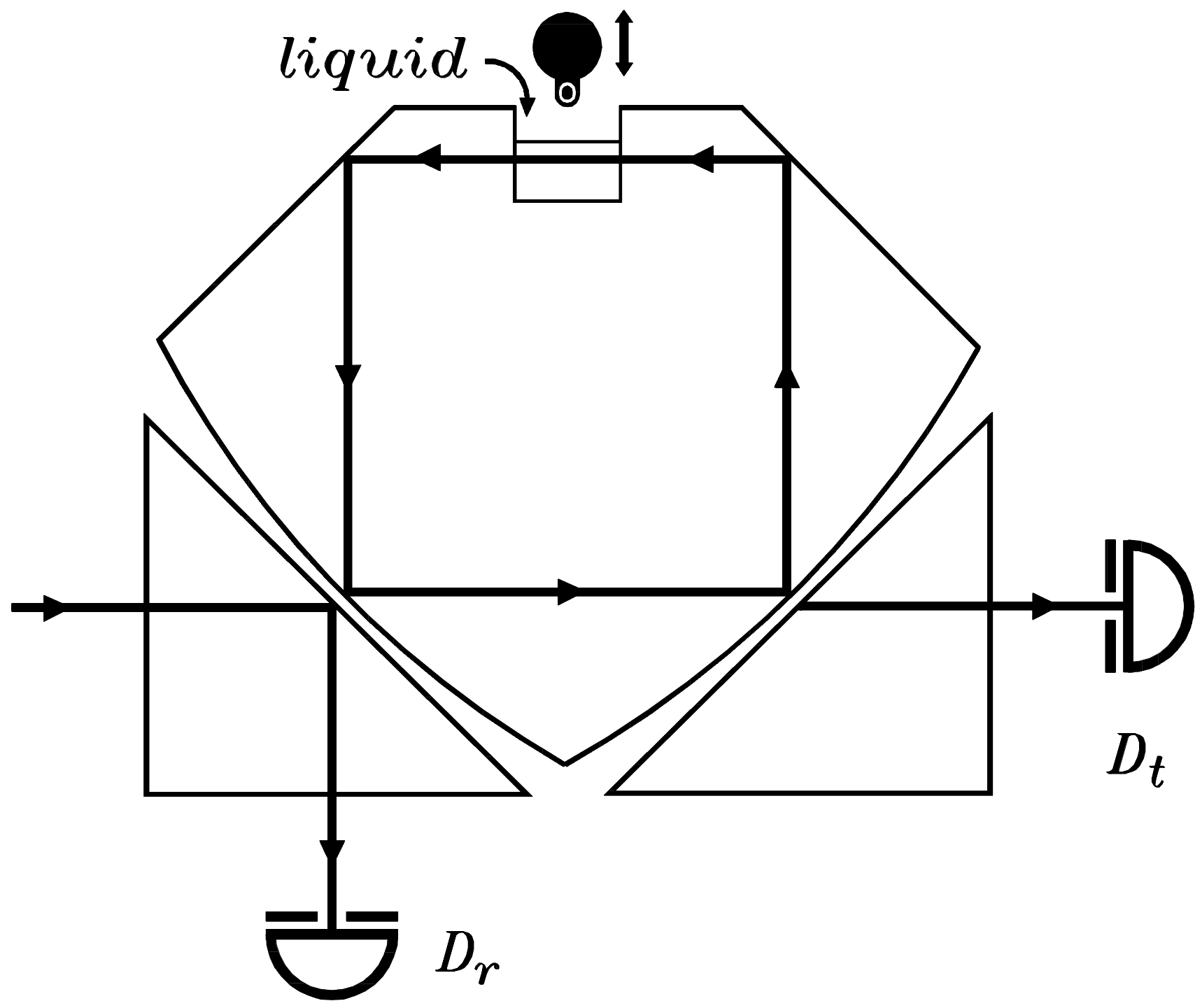

Fig. 1 


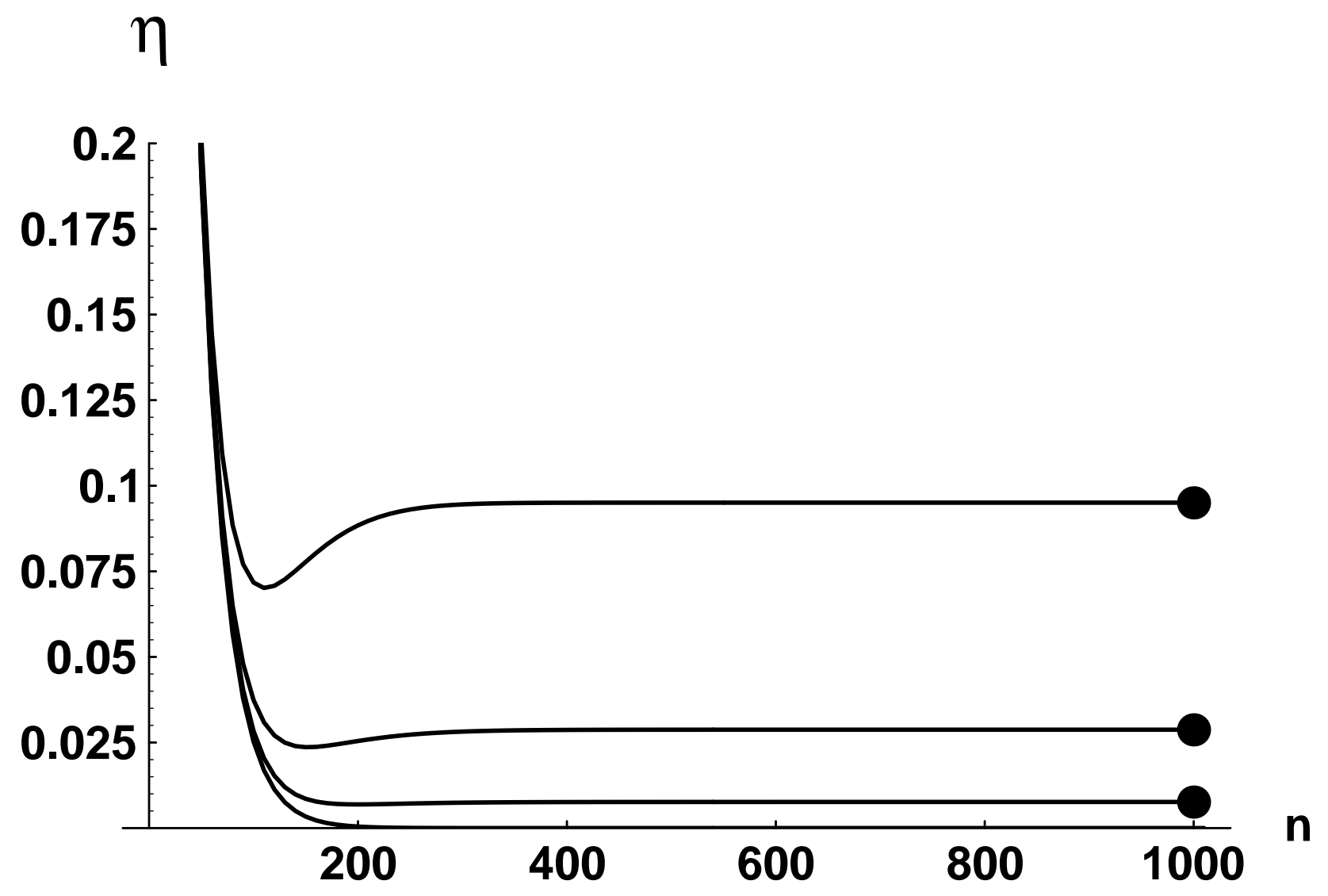

Fig. 2 
$\eta$

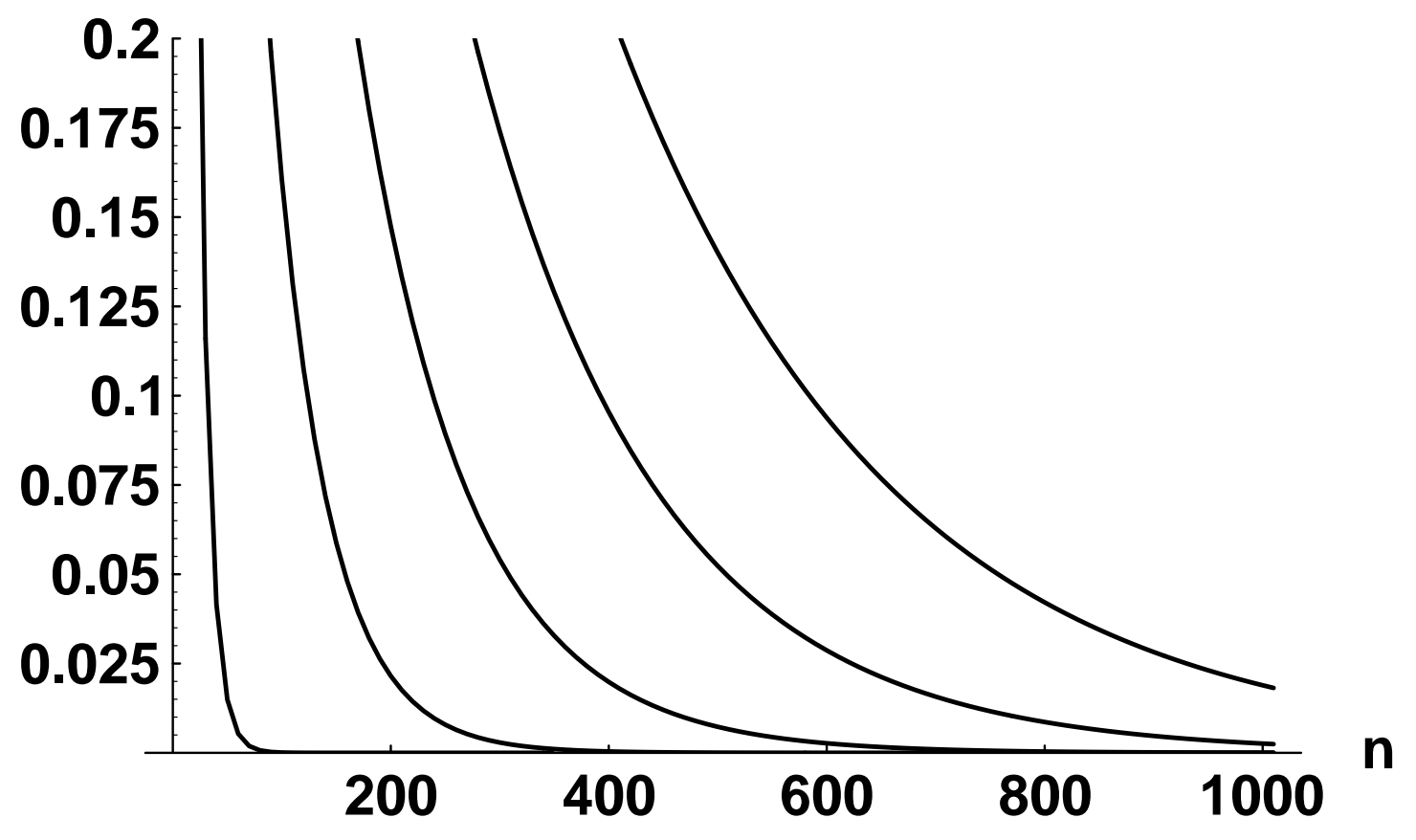

Fig. 3 


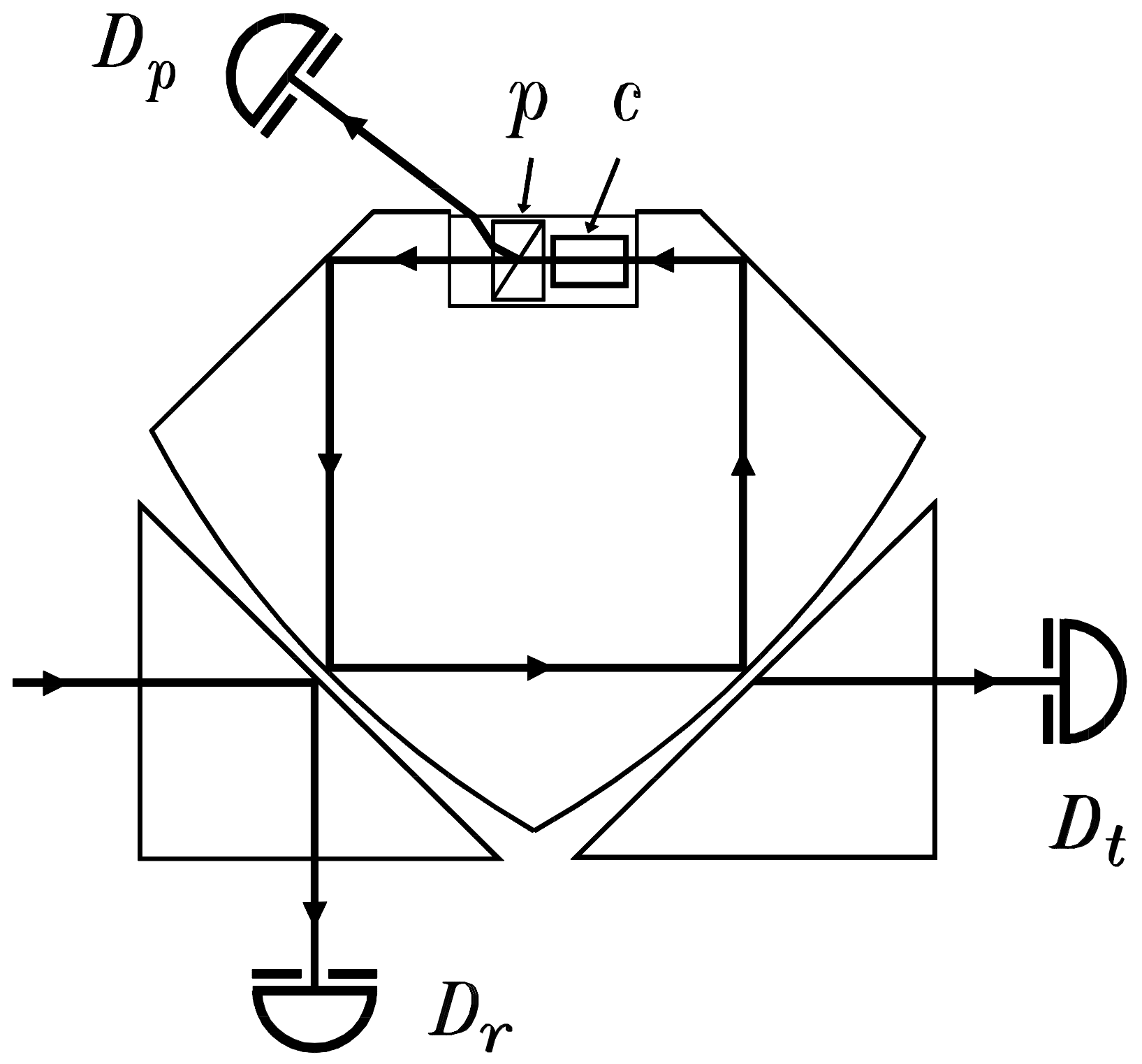

Fig. 4 